\title{
Using the Petri Network to Organize Resource Sharing between Two Processors
} Firas Mohammad Al-Abadi

College of Computer Science and Mathematics

University of Mosul, Iraq

\section{Received on: 12/10/2008}

\section{ABSTRACT}

Accepted on: 04/12/2008

With the development of information technology and the use of its applications as an essential aspect in almost all patterns of life especially in fields of analysis and real time systems, a problem of controlling resources appeared and how to provide the system with the resources at a proper time to avoid the system to be failed or vanishes, a state called a deadlock. This research aims to use the modern concept of Petri Net to provide synchronization and coordination between systems having more than one processor, sharing a specific resources at a same time. The research concentrates on capability of applying feed backing concept of Petri Net to achieve a good synchronization between different systems to work efficiently without being in deadlock.

Keywords: information technology, real time systems, Petri Net, processor, deadlock.

$$
\begin{aligned}
& \text { استخدام مفهوم شبكة بتري لتنظيم مشاركة المصادر بين معالجين } \\
& \text { فراس محمد العبادي }
\end{aligned}
$$

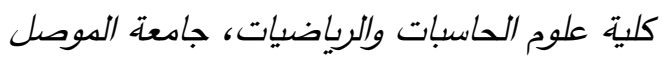

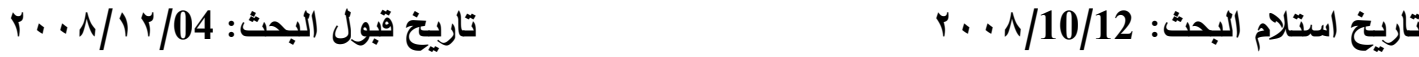

\section{الملخص}

مع التطور الحاصل في تقنية المعلومات واستخدامها كعامل أساسي وإشراكها في جميع أنماط الحياة وبشكل خاص في مجال التحليل وأنظمة الزمن الحقيقي ، ظهرت مشكلة السيطرة على المصادر وكيفية توفيرها

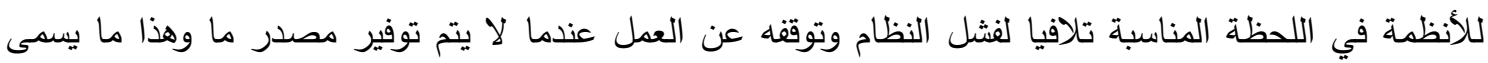
بمشكلة القفل الميت(Deadlock).

الهدف من هذا البحث هو استخدام مفهوم شبكة بتري (Petri Net) الحديث للعمل على توفير تزامن

وتتاسق بين الأنظمة التي تملك أكثر من معالج واحد وتثترك في العديد من المصادر فيما بينها بنغس الوقت. يركز البحث على إمكانية تطبيق مفهوم التغذية الراجعة (Feedback) على شبكة بتري للحصول على منى تزامن جيد بين الأنظمة لتعمل بشكل صحيح دون الوقوع في مشكلة القفل المميت. الكلمات المفتاحية: تقنية المعلومات، أنظمة الزمن الحقيقي، شبكة بتري، المعالج، القفل المميت.

تعتبر شبكة بتري أداة واعدة لثرح ووصف ودراسة أنظمة معالجة المعلومات وهي من الخصائص المتعلقة بالنظام (System) مثل التزامن (Synchronous) والتوزيع (Distributed) والتوازي (Parallel) والحالات [9][8]. (Stochastic) و الغير مستقرة (Nondeterministic) تم اكتثاف شبكة بتري من قبل Carl Adam Petri حيث بدأت مفاهيمها الجزئية في 1962 وتعتبر أداة نمذجة (Modeling Tools) أو نمذجة رياضية (Mathematical) أو مخطط توضيحي (Graphical) ويمكن أن تطبق على عدة أنظمة وهي أداة موثقة . 
يمكن استخدام شبكة بتري كاتصالات مرئية (Visual Communication) مشابه للمخطط الانسيابي (Flow Chart)

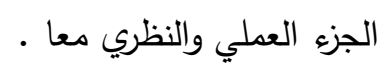

التعاريف العالمية لشبكة بتري عديدة فعلى سبيل المثال الجزه الأول من المقياس العالمي يقدم تعاريف

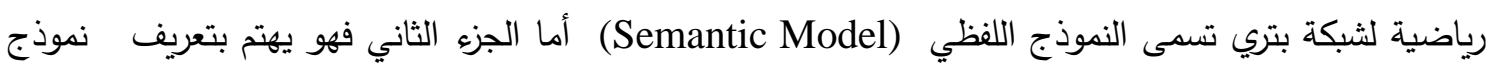

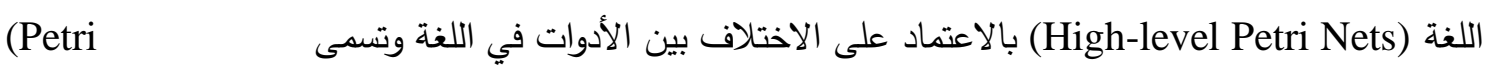
Net Markup Language (PNML)) هذا البحث من الشبكة. [7] هناك شبكة بتري يطلق عليها (Baukasten) والتي من شأنها دعم وتطوير البرامج والتطبيقات وهذا

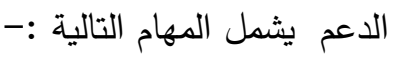

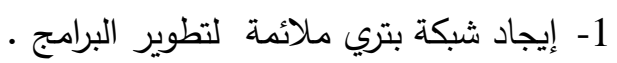
2- استخدام تقنية شبكة بتري التي تخدم ناحية تطوير التطبيقات . 3- إيجاد نوع مناسب من أنواع شبكة بتري ملائم للتطبيقات . 4- إيجاد الأداة المناسبة .

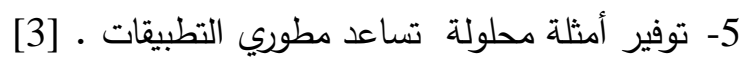
أهم المشاكل التي تحدث في الأنظمة والتي تعمل بالتزامن والتوازي هي مشكلة القفل المميت

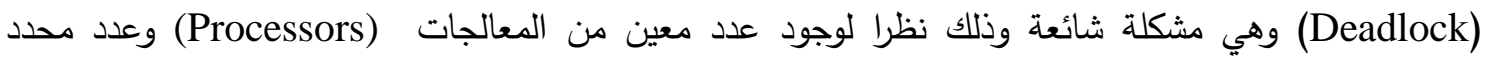
للمصادر (Recourses) مما يتطلب وجود مشاركة بالمصادر لغرض استمرار النظام في العمل بشكل صحيح وتام، هذه المشاركة بالمصادر من قبل المعالجات تتطلب استدعائها في زمن معين لاستخدامها وإرجاعها للاستفادة منها في معالجة أخرى، فماذا يحدث إذا ما تم طلب المصدر من قبل أكثر من معالج (Processor) في آن واحد؟ لتهن النتيجة بالتأكيد توقف النظام عن العمل بسبب عدم توفر المصدر في تلك اللحظة وهذا ما يسمى بمشكلة القفل من فئل

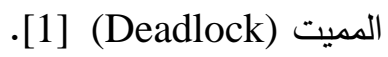

هناك في الحقيقة عدة طرق واستراتيجيات من شانها أن تعمل على حل مشكلة القفل المميت في الأنظمة

$$
\text { إما عن طريق :- }
$$

المنع (Prevent)

التجنب (Avoidance)

[11] التصحيح (Detection and Recovery)

كل طريقة من هذه الطرق ممكن أن تعالج مشكلة القفل المميت ولكن حسب اعتبارات عديدة منها إن كان المصدر قابل للمشاركة أم لا إي هل يسمح بمشاركة أكثر من معالج في نفس الوقت أم لا، كمثال على ذلك

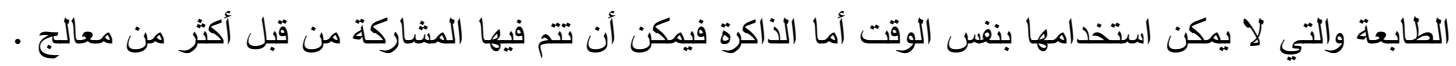

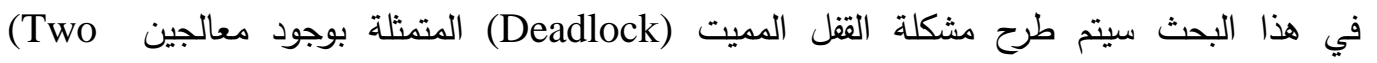
(Two Resources) تتم الششاركة فيما بينهما. 


\section{2. شبكة بتري Petri Net}

هي واجهة رسومية وأداة نمذجة رياضية، وهي تتألف من أماكن (Places) وانتقالات (Transition)

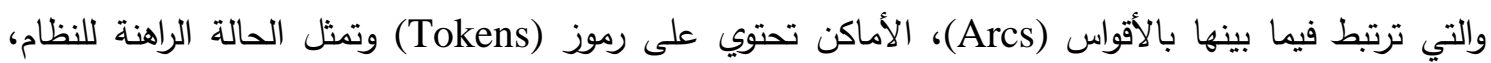

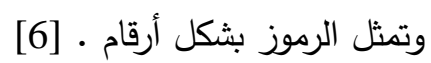

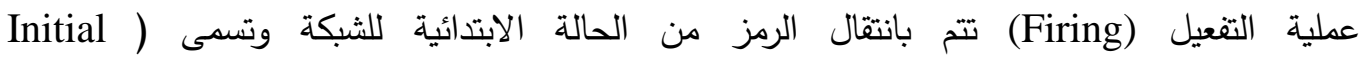
(Marking(M0) الأماكن والانتقالات كمفهوم شبكة بتري المستقلة وغير المستقلة. [2] تمثل الأماكن بدوائر والانتقالات بمربعات أو صناديق مرتبطة فيما بينها بأقواس وتعبر الأماكن عن الثرط (Conditions) والانتقالات هي الحدث (Event) [4] ، الجدول(1) يوضح بعض عمليات التغيير المثالية لعملية الانتقال.

الجدول(1) عمليات التغيير[4]

\begin{tabular}{|c|c|c|}
\hline Input Place & Transition & Output Place \\
\hline Precondition & Event & Post Condition \\
\hline Input Data & Computation Step & Output Data \\
\hline Input Signal & Signals Process & Output Signals \\
\hline Resources Needed & Task or Job & Resources Released \\
\hline Buffers & Processor & Buffers \\
\hline
\end{tabular}

2.1

شبكة بتري تتألف من خمسة رموز هي وهي مجموعة منتهية من الأماكن P = \{P1, P2, P3, ….....,Pm $\}$ T $=\{t 1, t 2, t 3, \cdots \cdots . . . ., t n\}$ F $=\subseteq(\mathrm{P} \times \mathrm{T}) \cup(\mathrm{T} \times \mathrm{P})$ وزن الدالة $\mathrm{W}=\mathrm{F} \rightarrow\{1,2,3, \cdots, \cdot, \mathrm{n}\}$ (Marked) بداية الوسم M0 = P $\rightarrow\{1,2,3, \cdots \cdots ., m\}$ $\mathrm{P} \cap \mathrm{T}=\varnothing$ and $\mathrm{P} \cap \mathrm{T} \neq \varnothing$ لوصف عملية الوسم في شبكة بتري هنالك قواعد للانتقال أو التقعيل وهي :-

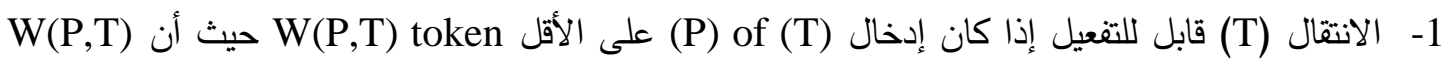

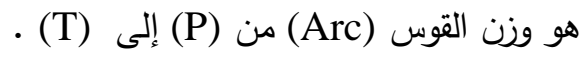
2- قابلية التفعيل (T) ممكن أن تحدث أو لا تحدث اعتمادا على ما مطلوب من النظام. 
3- عملية التفعيل هي قابلية تفعيل (T) وتحريك W(P,T) token جزيئا من كل مدخل (P) لـ (T) وإضافة

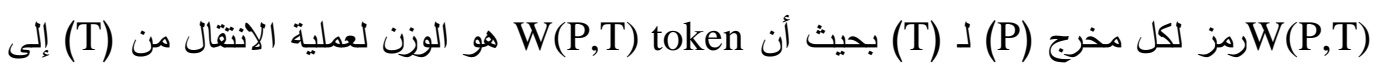

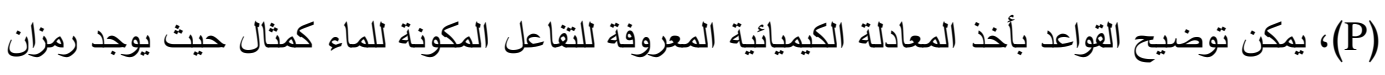
في كل واحد من الأماكن كما في المخطط التوضيحي(1)(أ) حيث تظهر جزيئتان من كل من الهدروجين

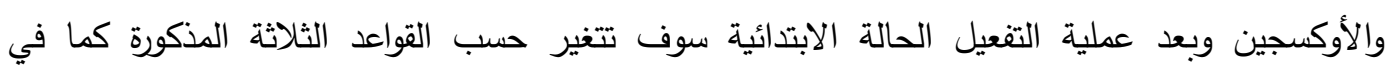
المخطط التوضيحي (1)(ب) وبعد)

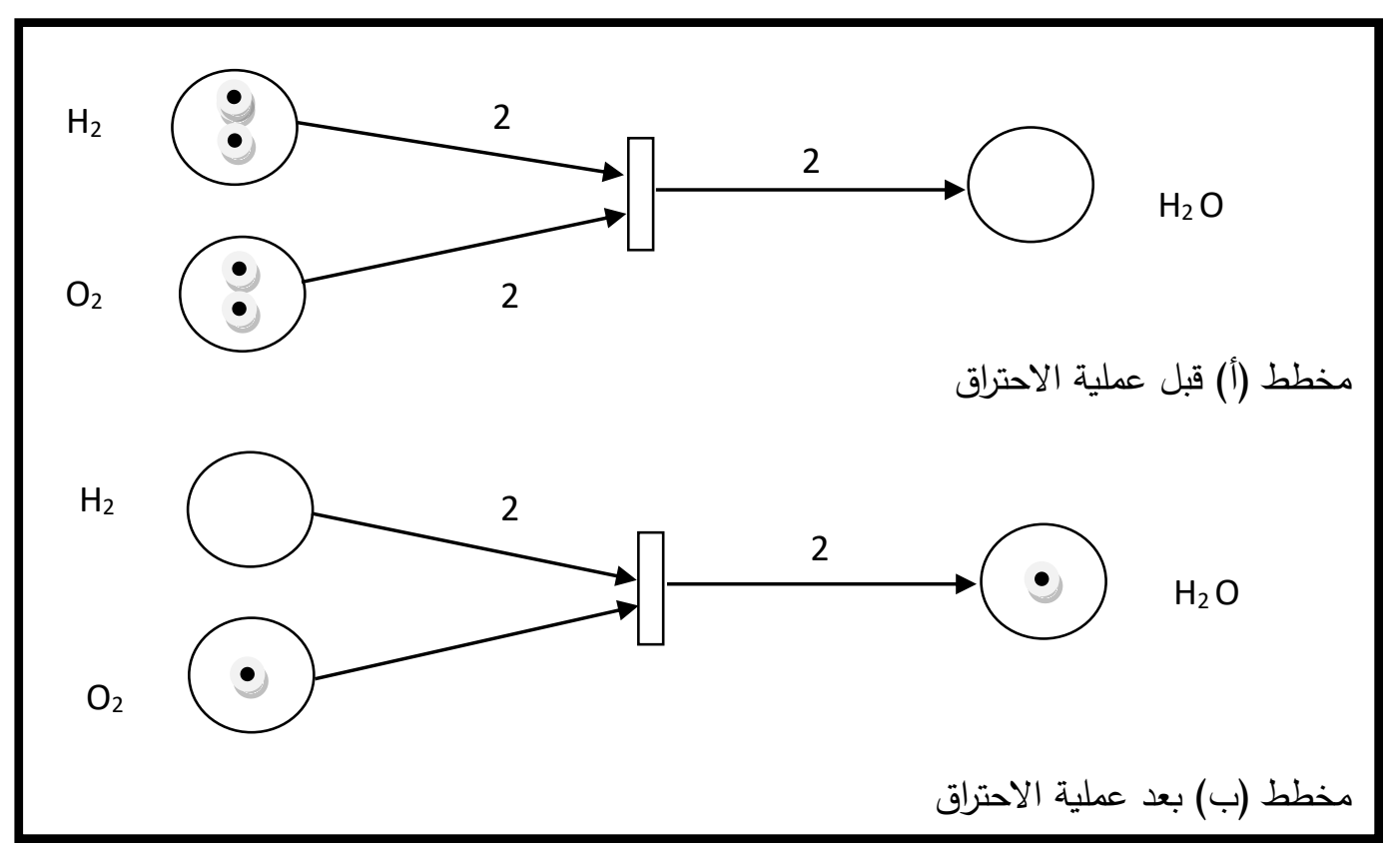

مخطط (1) توضيح معادلة الماء الكيميائية باستخدام Petri Net

والعملية سوف لن تحدث مجددا لعدم إمكانية تفعيل (T) مرة أخرى، وهناك مفهوم متعلق أيضا بشبكة

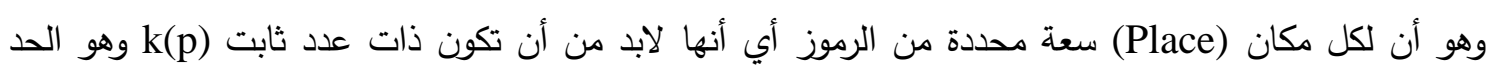

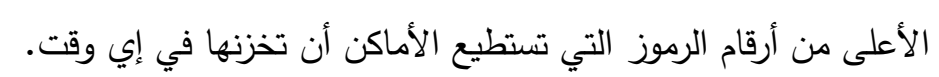

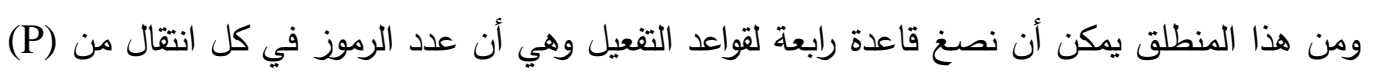
إلى (T) يجب أن لا يتجاوز سعة k(p) بعد عملية التفعيل. 3. تمثيل المسألة باستخدام شبكة بتري يمكن تمثيل مسألة النظام الذي يحوي على أكثر من معالج ويتطلب المشاركة في المصادر

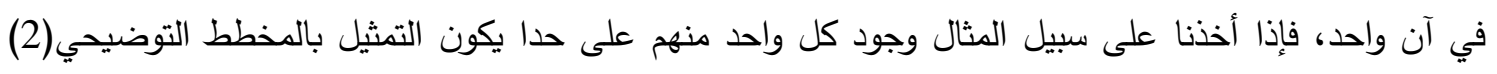



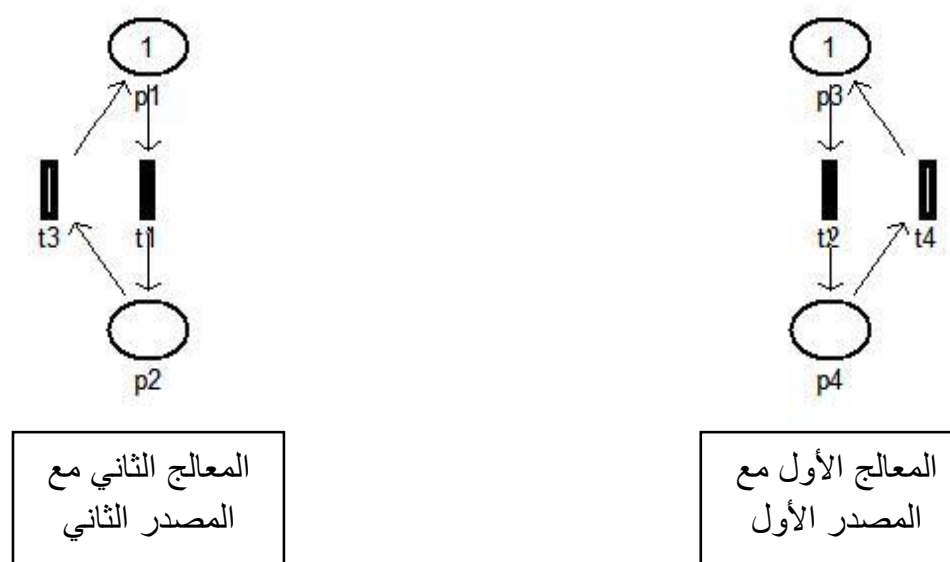

المعالج الأول مع المع المع

المصدر الأول الاول

\section{مخطط (2) يمثل معالجين مع مصدرين باستخدام Petri Net}

تم تمثيل المعالجين بـ P1,P3 وتمثيل المصدرين P2,P4 والرقم واحد داخل الدائرة هو الرمز والذي يمثل عملية البدء بطلب المصدر من قبل المعالج وأما (Transition) ، وهو ضروري ضمن مفهوم شبكة بتري لإتمام عملية التطبيق [5]، المخطط التوضيحي أعلاه

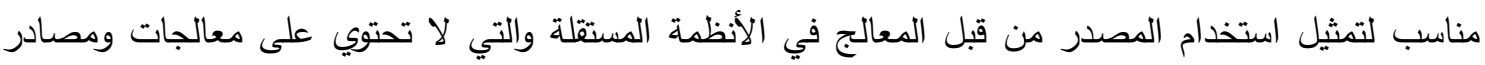
عدة مما يجنبها الوقوع في القفل المميت (Deadlock).

Petri Net 3.1

تمثيل العملية نفسها على أنظمة متعددة المعالجات فستكون كما في المخطط التوضيحي (3) التالي :-

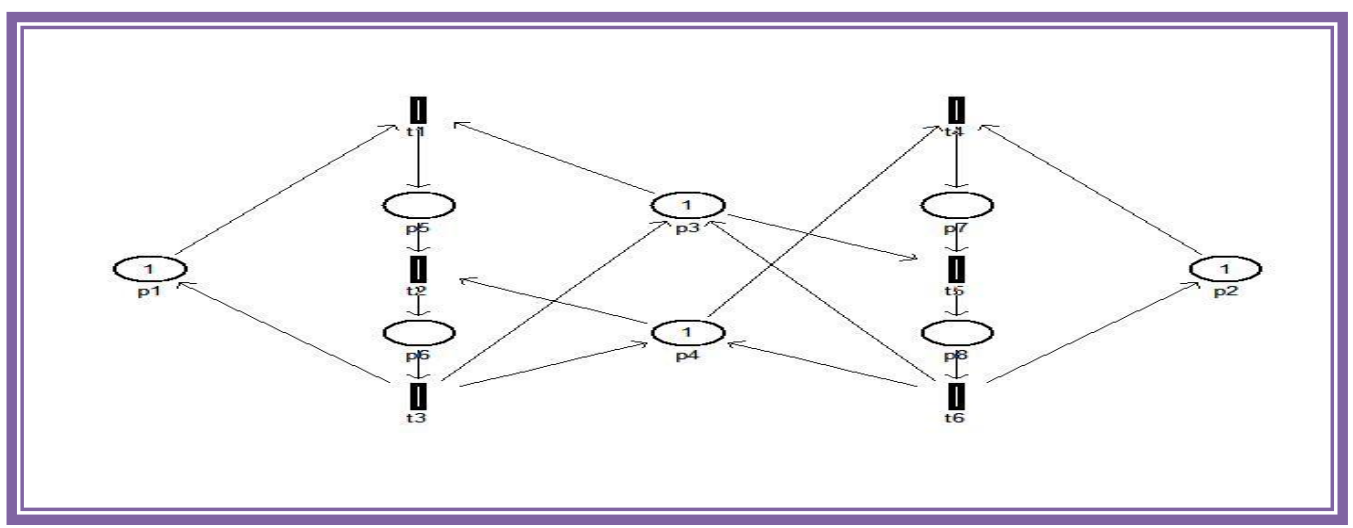

مخطط (3) عملية مشاركة المصدرين لكلا المعالجين

تم تمثيل النظام بمجموعة من الأماكن وهي والانتقالات \{t1,t2,t3,t4,t5,t6\} ولبدء عملية طلب المصدرين من قبل المعالجين في آن واحد تم إضافة رمز (Token) في كل من 


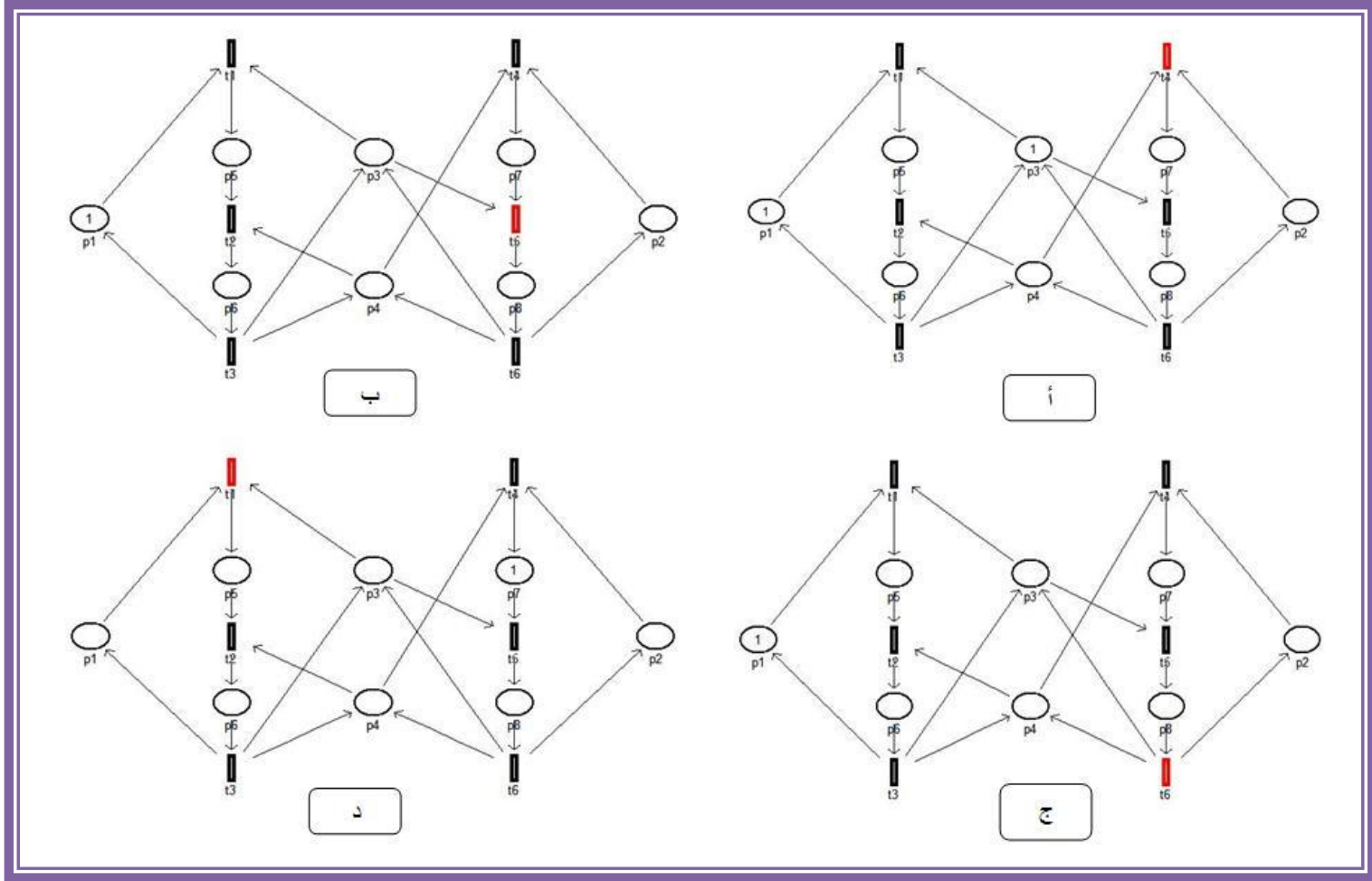

\section{firing مخطط (4) تنفيذ سلسلة من عمليات الـ}

عملية التفعيل ستتم إما في الـ t1 or t4 على حد سواء، وفيما يلي سلسلة من الخطوات باستخدام نموذج

$$
\text { شبكة بتري :- }
$$

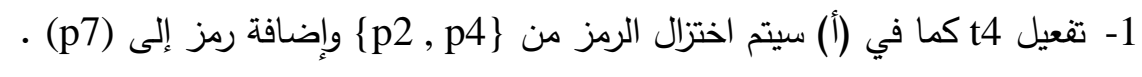

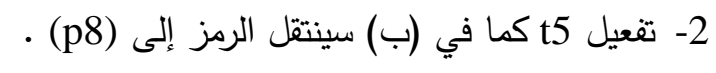

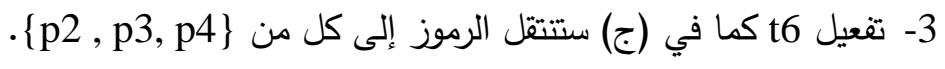

4- تفعيل t1 كما في (د) ستتنقل الرموز إلى (p5) و تفعيل t4 مرة أخرى كما في النقطة رقم(1) عندها

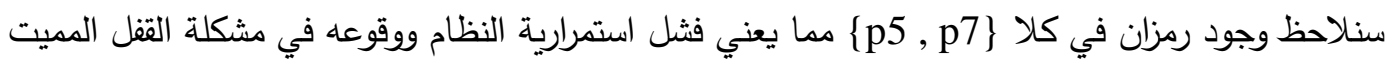
وذلك لعدم إمكانية تفعيل t5eadlock) في المخطط التوضيحي(5) التالي. 


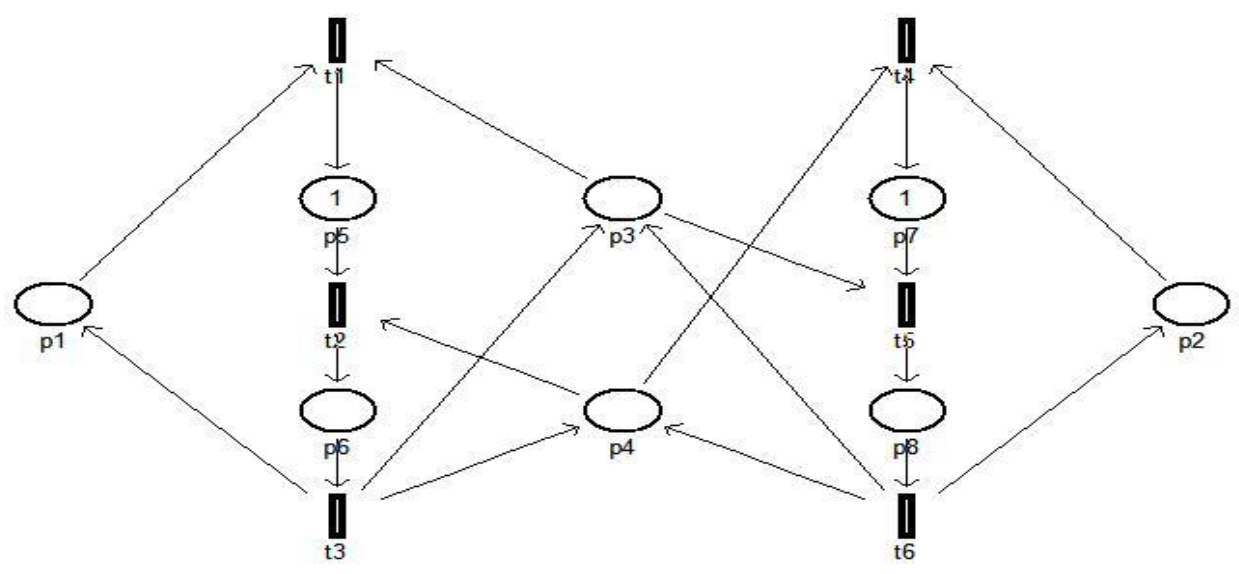

مخطط (5) يبين وقوع النظام في حالة الـDeadlock

فيما يلي جدول(2) إحصائية تمثل عملية اختزال وإضافة الرموز من الأماكن حيث :-

. t تمثل عدد مرات وقوع الحدث أي عدد مرات تفعيل الـ Event

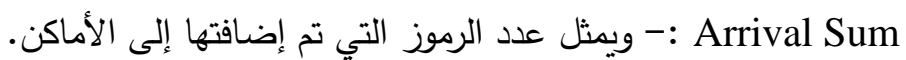
Throughput Sum : ويمثل عدد الرموز المختزلة من الأماكن.

جلول (2)إحصائية الـ Places خلال 5 Event

\begin{tabular}{|c|c|c|}
\hline Place Name & Arrival Sum & Throughput Sum \\
\hline \hline $\mathrm{p} 1$ & 0 & 1 \\
\hline $\mathrm{p} 2$ & 1 & 2 \\
\hline $\mathrm{p} 3$ & 1 & 2 \\
\hline $\mathrm{p} 4$ & 1 & 2 \\
\hline $\mathrm{p} 5$ & 1 & 0 \\
\hline \hline $\mathrm{p} 6$ & 0 & 0 \\
\hline \hline $\mathrm{p} 7$ & 2 & 1 \\
\hline \hline $\mathrm{p} 8$ & 1 & 1 \\
\hline
\end{tabular}

3.2 استخدام أسلوب الـ Lookhead Feedback للتخلص من مشكلة Deadlock يمكن الاعتماد على شبكة بتري في حل وتتظيم العديد من المسائل وذلك باستخدامها ومزاوجتها مع مانك التقنيات والأساليب المختلفة، وباستخدام أسلوب التغذية الراجعة وتطبيقها على شبكة بتري ستوفر إمكانية التخلص

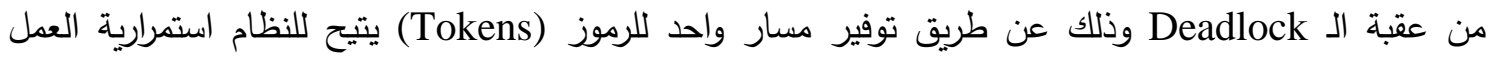
ويجنبه الفشل، هذا يعني استمرار النظام إلى مالا نهاية أو توقفه حسب برمجة النظام من قبل المستخدم كاستخدام

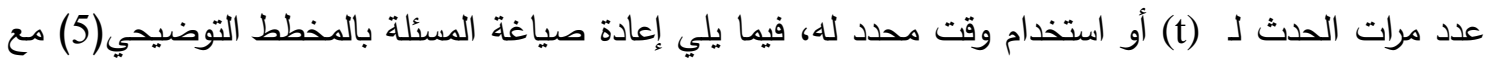
أسلوب التغذية الراجعة . 


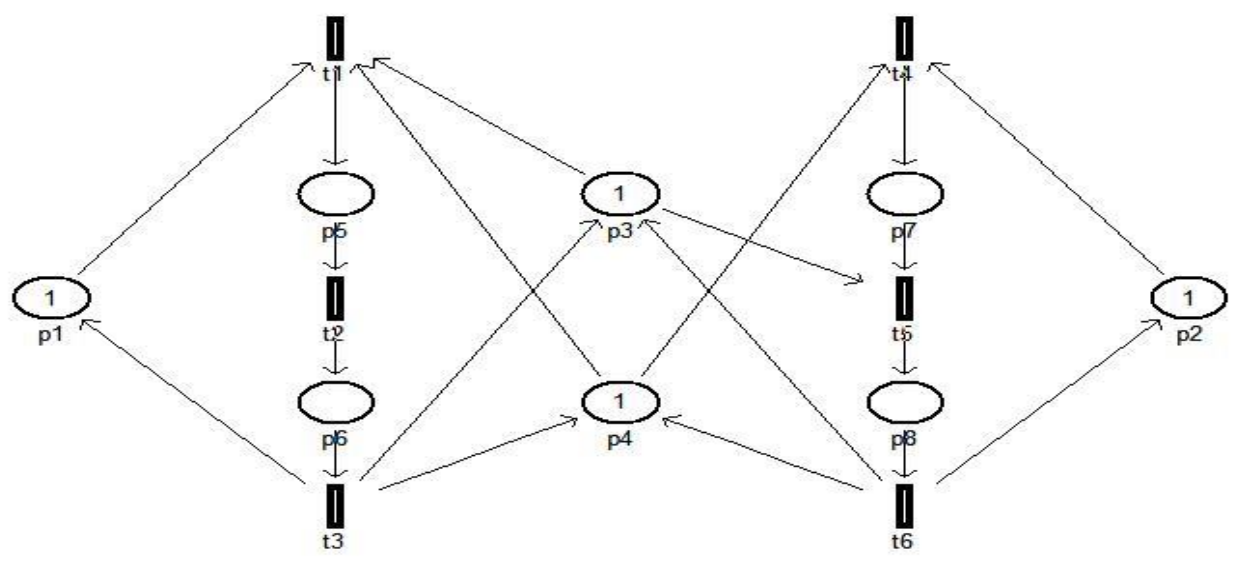

مخطط (5) استخدام أسلوب التغذية الراجعة للتخلص من الـ Deadlock

عملية التغذية الراجعة تمت عن طريق ربط قوس (arc) بين الـ p4 and t1 بدلا من الصيغة السابقة والتي كانت p4 and t2 وبهذا نجحنا في خلق توازن فعلي وعملي بين الطلبات من قبل المعالجين وتحديد بدقة عملية مشاركة المصدرين من قبل أي معالج دون الوقوع في حالة الققل المميت وهذه سلسلة من الأحداث تمت فئ فيها

استخدام المصدرين دون عائق ممثلة بمخطط توضيحي (6) أدناه (الأسهم المفردة تمثل الانتقالات المفعلة) . عملية التفعيل تتم أما في الـ t1 or t4 ، فيما يلي سلسلة خطوات باستخدام التغذية الراجعة في نموذج لثبكة بتري

$$
\begin{aligned}
& \text { 1- تفعيل t4 كما في (أ) سيتم اختزال الرموز من fp2, p4 وإضافة رمز إلى (p7) . }
\end{aligned}
$$

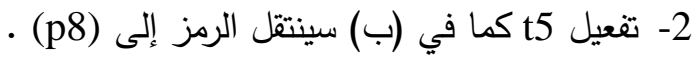

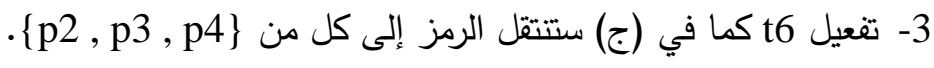

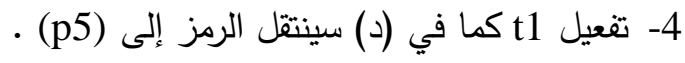

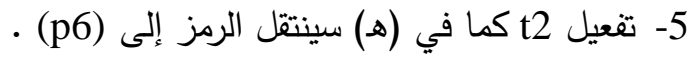

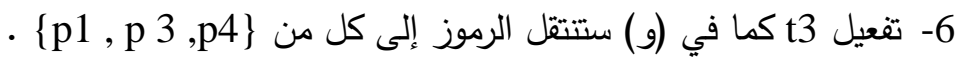



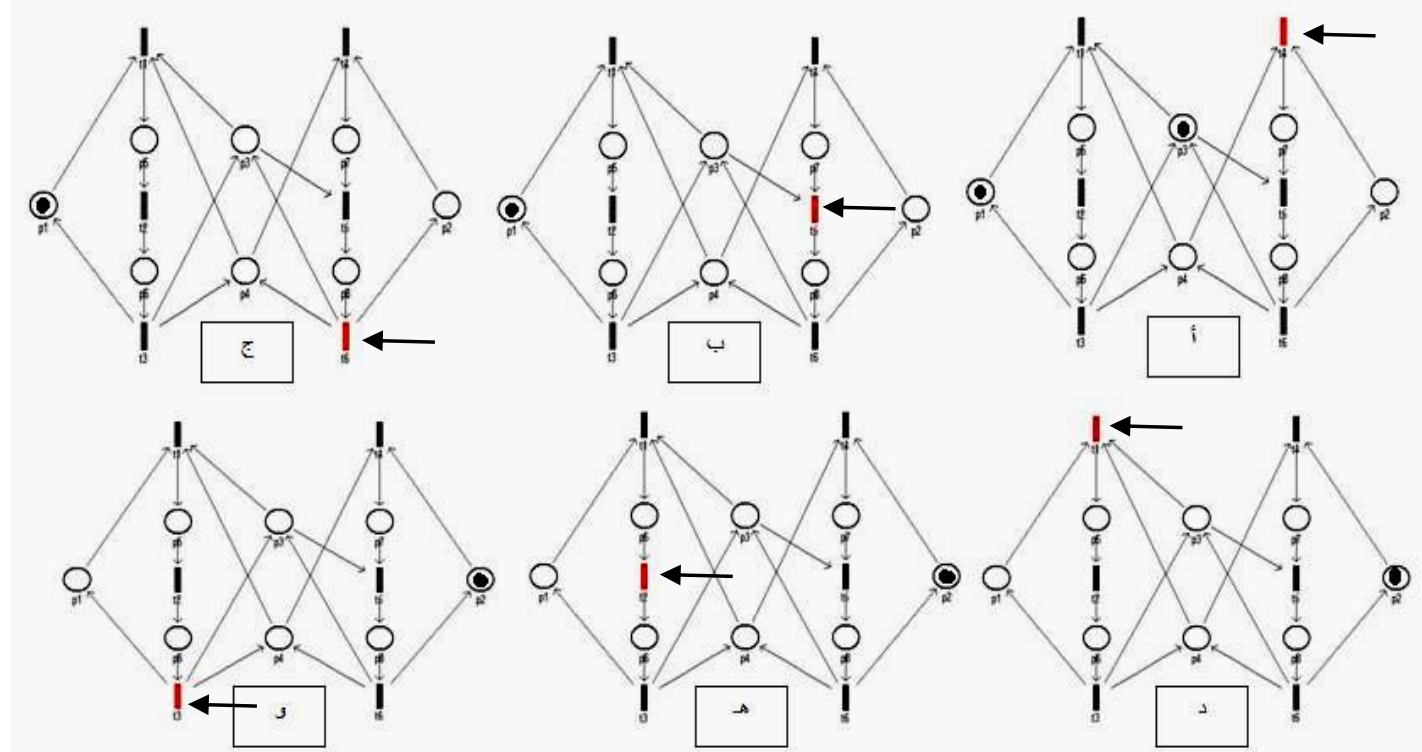

مخطط (6) سلسلة من عمليات الـ Firing باستخدام التغذية الراجعة

وهكذا سوف تعاد العملية من جديد بدأ من نقطة (1) إلى (6) دون حدوث تقاطع في عملية التتفيذ، فيما يلي جدول(3) إحصائية تمثل عملية اختزال وإضافة الرموز من الأماكن حيث أن عدد الأحداث (Event) هو دون

جدول(3)|إحصائية الـ Places خلال 10 Event

\begin{tabular}{||c||c|c||}
\hline Place Name & Arrival Sum & Throughput Sum \\
\hline $\mathrm{p} 1$ & 1 & 2 \\
\hline $\mathrm{p} 2$ & 2 & 2 \\
\hline $\mathrm{p} 3$ & 3 & 4 \\
\hline $\mathrm{p} 4$ & 3 & 4 \\
\hline $\mathrm{p} 5$ & 2 & 1 \\
\hline \hline $\mathrm{p} 6$ & 1 & 1 \\
\hline \hline $\mathrm{p} 7$ & 2 & 2 \\
\hline \hline $\mathrm{p} 8$ & 2 & 2 \\
\hline
\end{tabular}


4. - الخاتمة والاستنتاجات

إن الأنظمة التي تعاني من مشكلة القفل المميت (Deadlock) بسبب سوء إدارة استخدام المصادر الواجب توفرها لتنفيذ البرامج سواء أكانت مجموعة ملفات أو أجهزة إدخال وإخراج وغيرها، يمكن معالجتها بأسلوب يجعل من الاستحالة للنظام أن يقع في هذه المشكلة. نتيجة لتطبيق البحث تم التوصل إلى أن استخدام شبكة بتري في معالجة القفل المميت عن طريق دمج الثبكة بأسلوب التغذية الراجعة (Lookhead Feedback) أدى إلى نتائج ايجابية جعلت من استخدام مفهوم

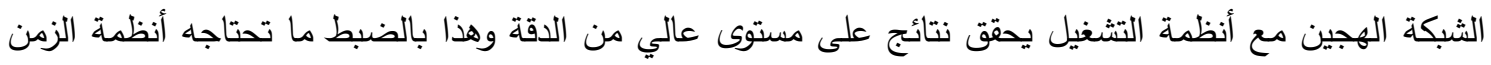
الحقيقي (Real Time System) من حساسية استخدام المصادر ودقة الوقت المطلوبة في هذه الأنظمة.

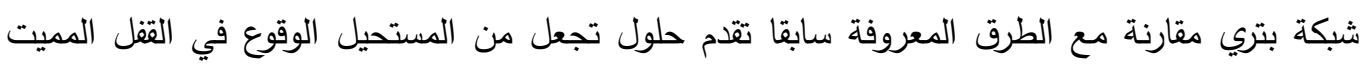

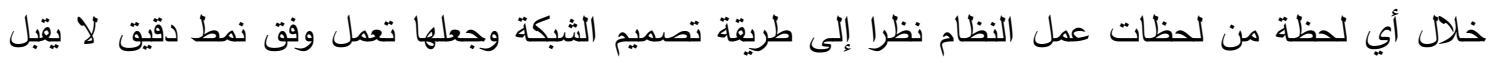
الخطأ كما هو موضح في المخططين السابقين المرقمين (6,5) ضمن الفقرة (3.2) . 


\section{ألمصادر}

[1] Commoner F. 'Deadlock in Petri Nets' Weak field Applied Data Research Inc, 1972.

[2] David R. , Alla H. , Discrete, 'Continuous, and Hybrid Petri Nets' publisher by Springer, 2005.

[3] Ehrig H. , Reisig W. , Rozenberg G., Weber H. , 'Petri Net Technology for Communication-based Systems', publisher by Springer, 2003.

[4] Galbo B. and et al., "Introductory Tutorial on Petri Nets", Petri Nets Conference, June 2000, Aarhus, Denmark.

[5] Holt A. W. and Commoner F., 'Event and Conditions', Applied Data Research Inc, 1970.

[6] Home Page 'http://www.daimi.au.dk/PetriNets.

[7] International Standard ISO/IEC 15909-2 WD Version 0.9.0, 'Software and Systems Engineering - High-level Petri Nets Part 2: Transfer Format', June 23, 2005.

[8] Magott J. 'Performance evaluation of concurrent systems using Petri Net', Inform. Processing Lett, 1984.

[9] MURATA T. 'Petri Nets Properties, Analysis and Applications' „FELLOW, IEEE, Invited Paper APRIL 1989.

[10] Petri C.A. , 'Kcommunication with Automata' , English translation NewYork, 1966.

[11] Silberschatz A., Galvin P. , Gagne, 'Operating System Concepts, fifth Edition', Addison Wesley Longman, 1998. 